\title{
An Exponential Growth in Incidence of Thyroid Cancer: Trends and Impact of CT Imaging
}

\author{
J.K. Hoang, K.R. Choudhury, J.D. Eastwood, R.M. Esclamado, G.H. Lyman, T.M. Shattuck, and X.V. Nguyen
}

\begin{abstract}
BACKGROUND AND PURPOSE: Workup of incidental thyroid nodules detected on CT imaging could be contributing to the increased diagnosis of small thyroid cancers. The purpose of this study was to evaluate recent trends in the incidence of thyroid cancer, and to determine the relationship between annual CT imaging volume and rate of thyroid cancer diagnosis.
\end{abstract}

MATERIALS AND METHODS: This retrospective cohort study used data bases for thyroid cancer and CT imaging volume. Thyroid cancer data from 1983-2009 were obtained from the Surveillance, Epidemiology, and End Results data base. National Council of Radiation Protection and Measurements Report No. 160 provided data on hospital and nonhospital CT imaging volume for 1993-2006. Trends in thyroid cancer were modeled for overall incidence on the basis of patient age, tumor histologic features, and tumor size and stage. Linear regression analysis was performed to evaluate the strength of the relationship between annual CT scan volume and the incidence of thyroid cancer by tumor size and histologic type.

RESULTS: In 2009, the incidence of thyroid cancer was 14 per 100,000, which represented a 1.9-fold increase compared with 2000 . The growth in incidence was exponential compared with a minimal linear increase in thyroid cancer mortality rate. The subgroup with the greatest change was subcentimeter papillary carcinoma, with doubling in incidence approximately every 6.2 years. The linear relationship between annual CT scan volume and the incidence of subcentimeter papillary carcinoma was very strong $\left(R^{2}=0.98 ; P<.0001\right)$.

CONCLUSIONS: The incidence of subcentimeter papillary carcinoma is growing at an exponential rate without significant change in mortality rate. The strong linear relationship between new cases of subcentimeter papillary carcinomas and the number of CT scans per year suggests that an increase in CT scans may increase the detection of incidental thyroid cancers.

ABBREVIATIONS: NCRP = National Council on Radiation Protection; SEER = Surveillance, Epidemiology, and End Results

T he incidence of thyroid cancer is increasing in the United States. A study published by Davies and Welch ${ }^{1}$ found that from 1973-2002, the incidence of thyroid carcinoma more than doubled. They reported that $87 \%$ of the increase in discovered cancers was attributable to tumors that were $2 \mathrm{~cm}$ or smaller, and despite earlier diagnosis, there was no change in mortality. These results provided compelling evidence for an "apparent" increase in cancer derived from increased use of diagnostic imaging tests,

Received May 31, 2013; accepted after revision August 5.

From the Departments of Radiology (J.K.H., K.R.C., J.D.E.), Radiation Oncology (J.K.H.), and Pathology (T.S.), Division of Otolaryngology Head and Neck Surgery (R.M.E.), and Department of Medicine (G.H.L.), Division of Oncology, Duke University Medical Center, Durham, North Carolina; and Department of Radiology (X.V.N.), The Ohio State University Wexner Medical Center, Columbus, Ohio.

Please address correspondence to Jenny K. Hoang, Department of Radiology, Division of Neuroradiology, Duke University Medical Center, Box 3808, Erwin Rd, Durham, NC 27710; e-mail: jennykh@gmail.com; jennykh@gmail.com

三 Indicates article with supplemental on-line appendix.

http://dx.doi.org/10.3174/ajnr.A3743 rather than a true increase in the biologic occurrence of thyroid cancer. This pattern of increased diagnosis of a silent reservoir of disease with an indolent natural history is similar to the epidemiologic changes in prostate cancer that occurred with prostaticspecific antigen and digital rectal examination-based screening. ${ }^{2}$ Davies and Welch ${ }^{1}$ and McLeod et $\mathrm{al}^{3}$ proposed that advances in sonography and fine-needle aspiration were leading to an increased diagnosis of subclinical (ie, small, impalpable) thyroid cancers that would otherwise remain asymptomatic during a patient's lifetime and not increase mortality rates. Epidemiologists have labeled this situation "overdiagnosis." 1,4

In recent years, 2 factors may have strongly influenced the work-up of subclinical thyroid nodules and, thus, the incidence of thyroid cancer. First, several societies, including the Society of Radiologists in Ultrasound and American Thyroid Association, published sonographic guidelines for biopsy of thyroid nodules on the basis of best available evidence and expert opinion. ${ }^{5,6}$ These societies raised concern that their recommendations could 
increase the biopsy rate of thyroid nodules and the incidence of thyroid cancer. ${ }^{5}$ Second, the use of diagnostic imaging has changed substantially in recent years. In particular, the use of CT has increased rapidly in the United States, at a rate greater than sonography imaging. ${ }^{7-9}$ A study of nationwide emergency department imaging use from 2000-2008 found that the use of sonography increased by $95 \%$, whereas CT increased by $227 \%$. In addition, CT made up 29\% of emergency department imaging, but sonography comprised only $4 \%$. CT scans of the neck, cervical spine, and/or chest can include the thyroid gland and may be a source for the detection of incidental thyroid nodules that subsequently receive work-up and biopsy.

The aim of our study was to evaluate the recent trends in the incidence of thyroid cancer, and to determine the relationship between annual CT imaging volume and thyroid cancer incidence. Our hypothesis was that the incidence of subclinical thyroid cancers has continued to increase, and that the increase in thyroid cancers correlates strongly with the volume of CT imaging.

\section{MATERIALS AND METHODS}

This retrospective cohort study evaluated 2 large data bases for thyroid cancer and CT imaging volume. The study was approved by our institutional review board.

\section{Data Sources and Analysis}

Data on thyroid cancer incidence and survival for thyroid cancer were obtained from the National Cancer Institute's Surveillance, Epidemiology, and End Results (SEER) data base. The SEER program collects cancer data from multiple population-based registries, with the 9 registries that were active throughout this interval comprising $10 \%$ of the US population. Available data from 19832009 included patient sex, age, and follow-up, as well as tumor size, histologic features, and staging.

Data on CT imaging volume were obtained from the National Council on Radiation Protection (NCRP) and Measurements Report No. $160 .{ }^{10}$ The report provides the annual number of CT procedures in both hospital and nonhospital facilities in the United States from 1993-2006.

\section{Statistical Analyses}

Trends in Incidence and Mortality. Trends in thyroid cancer were modeled for overall incidence and mortality. Incidence was also modeled as a function of patient age, as well as tumor histologic features, size, and stage, to determine the characteristics of the tumors that are increasing in incidence. The number of new cases annually was assumed to have a Poisson distribution, and a multivariate log-linear model was proposed for the mean incidence to determine variables influencing the rate of change in incidence (On-line Appendix A). The models were fitted based on maximal likelihood by use of iteratively reweighted least squares.

Relationship of CT Imaging and Thyroid Cancer. Trends in CT volume were modeled to describe growth in CT as an exponential function of time, fitted by log-linear regression. The slope of the fitted regression line is interpreted as the annual rate of increase in CT volume.
We fit a linear regression model to evaluate the relative impact of annual CT imaging volume on the incidence of thyroid cancer for 1993-2006, as influenced by tumor size and histologic appearance. A linear regression model was chosen because we aimed to describe the dependence of new cases of thyroid cancer on the number of CT scans for matched years. If incidental nodules seen on CT led to a diagnosis of thyroid cancer, then we would expect cancer incidence to increase proportionately with CT scans.

The model was as follows:

$$
y_{s}(t)=\mu+\mu_{s}+b c(t)+b_{s} c(t)+\varepsilon_{s}(t)
$$

where $y_{s}(t)$ is the number of cases of thyroid cancer of size $s(<10$ $\mathrm{mm}, 10-14 \mathrm{~mm}, 15-19 \mathrm{~mm}, 20-29 \mathrm{~mm}, 30-39 \mathrm{~mm}$, or $>40$ $\mathrm{mm}$,) in year $t$ and $c(t)$ is the total number of CT scans in year $t$. The model explains the number of cases in $\mu$, the baseline number of cases of size $<10 \mathrm{~mm}$ in 1993, $b$, the rate of increase in cases per million extra CT cases for size $<10 \mathrm{~mm}, \mu_{s}$, the change in number of cases of size $s$ (relative to baseline) in 1993, and $b_{s}$, the change in rate of increase for cases of size $s$ (relative to baseline). The slope of the model (b) for tumor size was of particular interest because we would expect the slope to be higher for smaller cancers if incidental nodules seen on CT are contributing to the increase in diagnosis of thyroid cancer.

Statistical analyses were performed with SeerStat 7.1.0 (National Cancer Institute, Bethesda, Maryland) and $\mathrm{R}$ package (www.r-project.org). For all tests, a $P$ value $<.05$ was considered statistically significant.

\section{RESULTS}

\section{Trends in Incidence and Mortality}

A total of 52,930 cases of thyroid cancer were diagnosed in the 9 SEER geographic areas from 1983-2009. In 2009, the incidence of thyroid cancer was 14 per 100,000, which was a 1.9-fold increase compared with the year 2000 (Table 1). The trend in thyroid cancer incidence during this period seems to be exponential (Fig 1). By contrast, the trends in mortality and population growth seem approximately linear (Fig 1) and relatively much slower during the same period.

\section{Variables Influencing Trends}

Papillary carcinoma was the most common histologic type, comprising $88 \%$ of thyroid cancers in 2009 (Table 1) and having an exponential rate of growth in incidence (Fig 1). An exponential model was able to explain $96 \%$ of the variability (null deviance) in the data, suggesting an excellent fit (On-line Appendix B). The variable that most significantly influenced the increase in incidence was tumor size $<10 \mathrm{~mm}$. For papillary carcinoma with size $<10 \mathrm{~mm}$, the growth in incidence per year was $11.8 \%$, which translates into a doubling in incidence every 6.2 years (Fig 2).

Baseline rates of growth in incidence for other histologic types were considerably lower: follicular, 3.8\% (95\% CI, 2.4-5.1); medullary, 4.0\% (95\% CI, 1.2-6.6); and anaplastic, 1.5\% (95\% CI, $-21 \%$ to $18 \%)$.

\section{Relationship of CT Imaging and Thyroid Cancer}

The rate of increase in CT scan volume per year was not linear. From 1993-1999, the number of CT scans per year increased from 18.3 to 30.6 million per year (12.3-million increase). In the same 
Table 1: Thyroid carcinoma overall incidence and mortality, stratified by patient sex and tumor characteristics

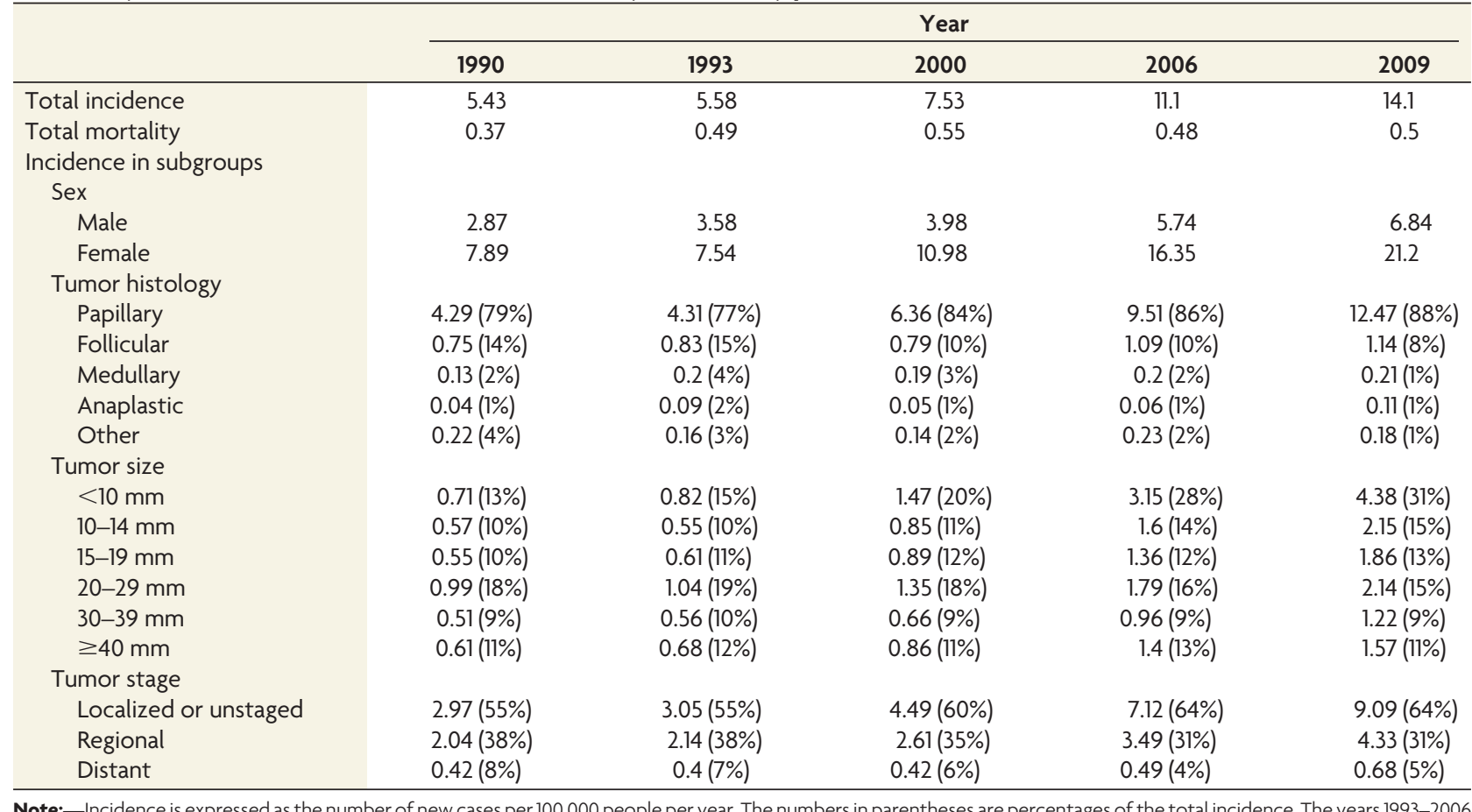

Note:- Incidence is expressed as the number of new cases per 100,000 people per year. The numbers in parentheses are percentages of the total incidence. The years $1993-2006$ represent the period for which annual CT volume data are also available.

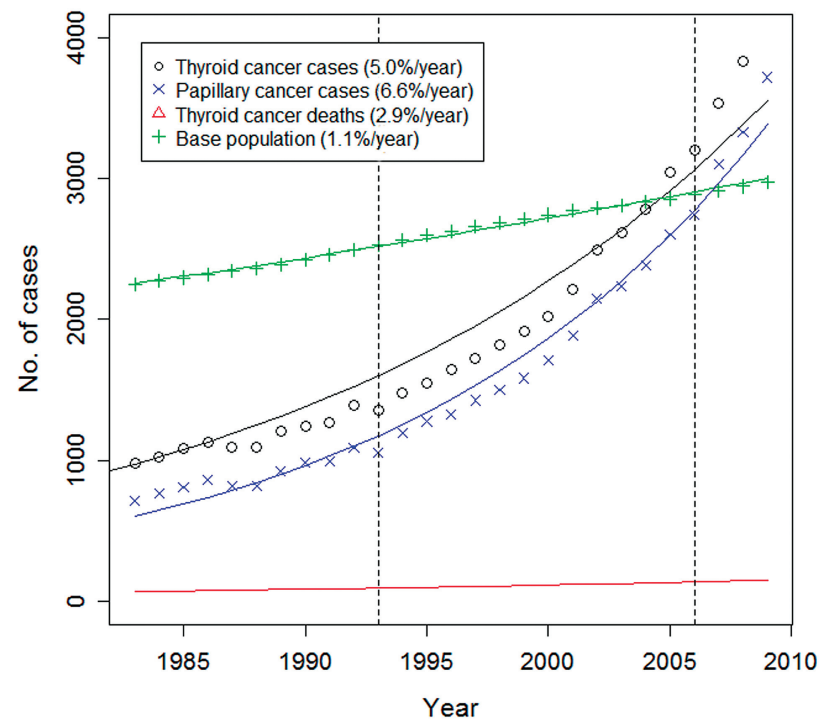

FIG 1. Trends in incidence and mortality of thyroid carcinoma. The rate of increase in incidence (\%) per year is shown for thyroid cancer, papillary cancer, thyroid cancer deaths, and the size of the population. The base population is expressed in units of persons $\times 10^{-4}$. All of the trend lines were fitted to an exponential function, but the low growth rates for base population and thyroid deaths could be approximated by linear growth curves. Dashed vertical lines indicate the period of 1993-2006 for which annual CT volume data were also available.

period from 2000-2006, CT volume per year had increased from 34.9 to 62.0 million, which represented an increase of 27.1 million scans. This finding represents a growth in CT scans of $10 \%$ per year.

The NCRP report did not provide the proportion of studies for each body region by year but did include the proportion of CT procedures for various body categories for 3 selected years for its 4

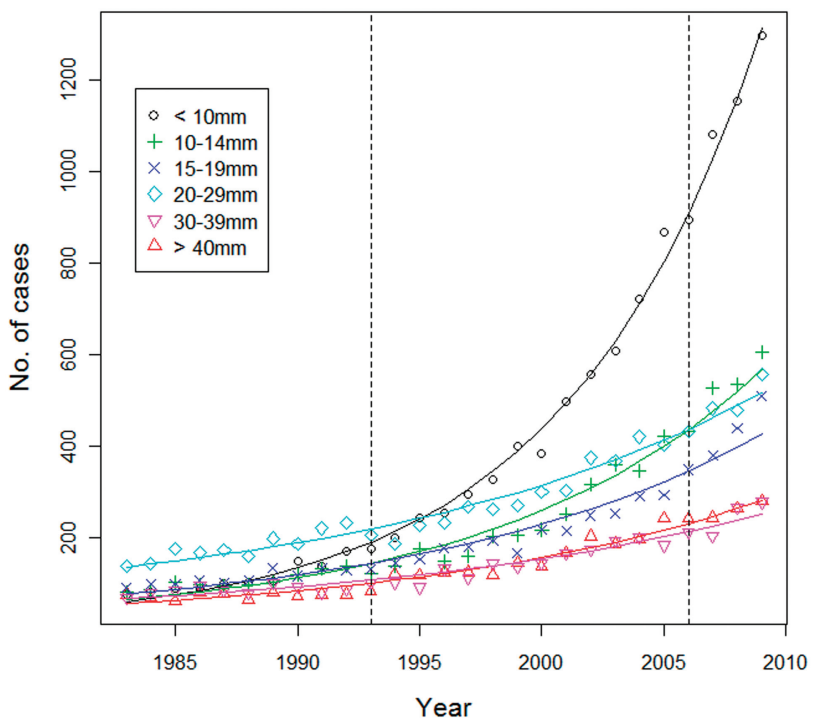

FIG 2. Trends in the number of new cases per year of papillary carcinoma by size. Symbols show observed values. Lines show fit from multivariate log-linear model for incidence (On-line Appendix B). Dashed vertical lines indicate the period of 1993-2006 for which annual CT volume data were also available.

largest datasets (commercial [IMV Benchmark report], Medicare, Veterans Affairs, and Large National Employer Plan). The IMV Benchmark report data represented the largest contribution to the data base (75\% of CT scans in 2003). According to this dataset, the proportion of studies attributed to head and neck and chest imaging from 1998 to 2006 was stable. Head and neck CT in 1998, 2003, and 2006 comprised 32\%, 31\%, and 29\% of CT studies, respectively. Chest CT in 1998, 2003, and 2006 comprised 17\%, $16 \%$, and $16 \%$ of CT studies, respectively.

Linear regression analysis of new cases of thyroid cancer and 
Table 2: Estimated coefficients for linear regression model for papillary thyroid cancer incidence

\begin{tabular}{lcccc}
\hline & Estimate & Std. Error & $T$ Value & $\operatorname{Pr}(<|\mathbf{t}|)$ \\
\hline Baseline intercept $^{\mathrm{a}}$ & 178.79 & 9.05 & 19.75 & $<.0001$ \\
Baseline slope CT $^{\mathrm{b}}$ & 15.6 & 0.39 & 39.84 & $<.0001$ \\
Diff. intercept size $^{\mathrm{c}}$ & & & & \\
10-14 mm & -52.93 & 12.8 & -4.13 & .0001 \\
$15-19 \mathrm{~mm}$ & -37.7 & 12.8 & -2.94 & .0043 \\
20-29 mm & 29.41 & 12.8 & 2.3 & .0245 \\
30-39 mm & -74.14 & 12.8 & -5.79 & $<.0001$ \\
>40 mm & -77.87 & 12.8 & -6.08 & $<.0001$ \\
Diff. slope CT:size & & & & \\
10-14 mm & -12.47 & 0.55 & -22.52 & $<.0001$ \\
15-19 mm & -8.72 & 0.55 & -15.75 & $<.0001$ \\
20-29 mm & -11.5 & 0.55 & -20.77 & $<.0001$ \\
30-39 mm & -10.28 & 0.55 & -18.57 & $<.0001$ \\
$>40 \mathrm{~mm}$ & -13.12 & 0.55 & -23.69 & $<.0001$ \\
\hline
\end{tabular}

Note:-The estimated coefficients indicate how changes in CT imaging volume, tumor size, and interaction between these 2 factors would affect thyroid cancer incidence compared with the baseline number of cases of size $<10 \mathrm{~mm}$ in 1993.

a Baseline intercept term is the estimated incidence in the year 1993.

b Baseline slope CT refers to the rate of increase in cases per million CT scans for tumors $<10 \mathrm{~mm}$ since 1993

'Diff. intercept size indicates how much the intercept changes for other sized tumors.

${ }^{d}$ Diff. slope CT:size indicates how much the slope changes for other sized tumors. A negative estimate value indicates that the intercept or slope would be lower than for the baseline case of size $<10 \mathrm{~mm}$.

CT volume by year demonstrated that the model fit the data very well. $R^{2}$ represents the proportion of variability of the data that could be explained by linear regression. For papillary cancers, the $R^{2}$ was 0.98 , whereas for other cancers, the $R^{2}$ was 0.96 . Our interest is primarily in the slope of the fitted lines, ie, the rate of increase in incidence per million CT scans. The highest rate of increase was for papillary tumors $<10 \mathrm{~mm}$ in size (Table 2). Slopes for all other sizes were 2.3-6.2 times lower (Table 2). For papillary cancers, the rate of growth in incidence (slopes) generally decreased with increase in size (Table 2 and Fig 3A). For nonpapillary cancers, the picture is the opposite: if anything, slopes increased with higher sizes (Fig 3B).

\section{DISCUSSION}

Although it took 30 years for the incidence of thyroid cancer to double before 2002, ${ }^{1}$ we found that the number of new diagnoses nearly doubled between the years 2000 and 2009 without significant change in mortality rate. Our observation that subcentimeter, and hence impalpable, thyroid cancers are growing at the greatest rate agrees with a body of literature that proposes imaging could be leading to the increased diagnosis of small thyroid cancers. $^{1,11,12}$

The influence of imaging on the detection of thyroid cancer began with the use of sonography and sonography-guided fineneedle biopsy in the 1980s. The incidence continued to increase as sonography machines became more widely used in the clinician's office. ${ }^{13}$ This study generates a hypothesis that the current trends could also be related to CT imaging in addition to sonography. Our data show a parallel increase in the use of diagnostic CT and cases of thyroid cancer, especially for subcentimeter papillary carcinoma. Although regression analyses do not infer causality, the linear model fits very well and has led us to consider 2 scenarios in which CT scanning could lead to a diagnosis of thyroid cancer. The first is that an increase in CT imaging detects more incidental
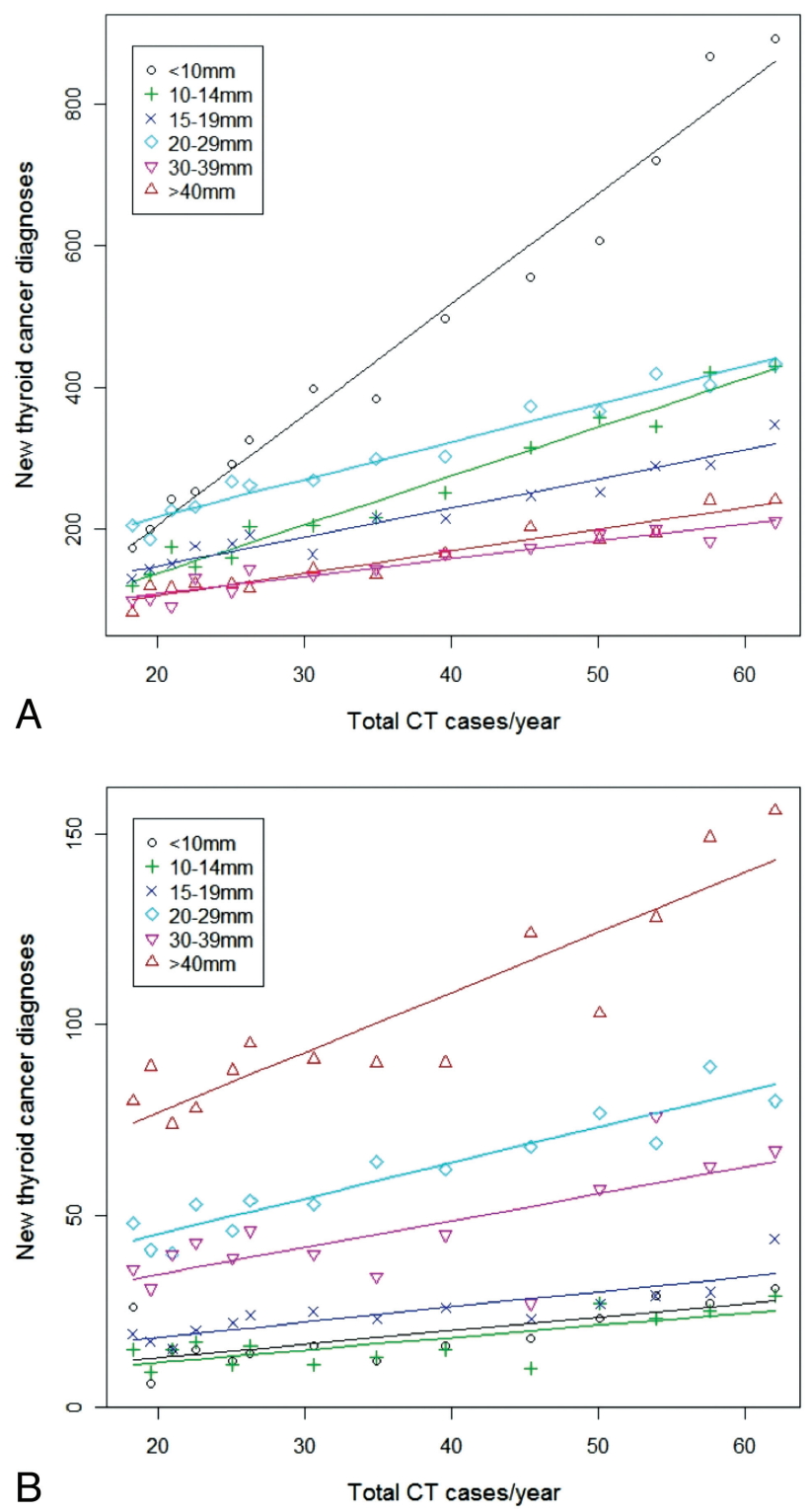

FIG 3. Relationship between annual CT scans and incidence of thyroid cancer for $(A)$ papillary carcinoma and $(B)$ nonpapillary carcinoma. The CT cases represents million cases per year.

thyroid nodules that receive work-up leading to the subsequent diagnosis of cancer. Alternatively, the microcarcinoma may not have been seen on the initial CT, but work-up of another nodule detected on CT could lead to detection of the microcarcinoma on thyroid sonography or in a diagnostic lobectomy surgical specimen. ${ }^{14}$

In our study, larger thyroid cancers $(>20 \mathrm{~mm})$ are also increasing, albeit at a lower rate than cancers $<10 \mathrm{~mm}$. Chen et $\mathrm{al}^{15}$ argued that diagnostic scrutiny from imaging would not account for the increase in larger thyroid cancers. We suggest that CT imaging could lead to the incidental diagnosis of larger cancers, as large nodules may be asymptomatic because of deep location in the posterior aspect of the thyroid or because of large body habitus. Wiest et $\mathrm{al}^{16}$ found that $52 \%$ of thyroid nodules $>2 \mathrm{~cm}$ were not palpable by experienced physician examiners. Furthermore, it is known from retrospective studies that incidental thyroid nod- 
ules can be $>2 \mathrm{~cm}$. Hobbs et $\mathrm{al}^{17}$ studied the presentation of thyroid nodules having sonography-guided biopsy, and found that nodules detected incidentally on imaging had a mean size of $26 \mathrm{~mm}$ (standard deviation, $17 \mathrm{~mm}$ ). In our study, the relationship between annual CT volume and the incidence of papillary carcinomas that were $>2 \mathrm{~cm}$ also fit the linear regression model well, but the rate of increase in incidence per million scans was 3-6 times lower than for subcentimeter papillary carcinoma.

It is also plausible that the relationship between CT and thyroid cancer is in reverse order to our main hypothesis: An increased incidence of thyroid cancer may be causing an increase in CT scans. The effect may represent use of CT for work-up of known thyroid cancer. Against this alternative hypothesis is that sonography is used far more frequently to evaluate newly diagnosed thyroid cancers than CT. CT scans are only obtained in newly diagnosed thyroid cancer for large tumors that may be invading the trachea or carotid artery, or have substernal extension. In fact, the American Thyroid Association strongly recommends against preoperative CT and MR imaging for thyroid cancer. ${ }^{6}$ The second argument against thyroid cancer causing an increase in CT use is the observation of the linear regression results for nonpapillary carcinoma. The linear regression slopes for different tumor sizes show a higher slope for larger nonpapillary cancers than smaller nonpapillary cancers, indicating that for a given CT scan, there are more larger cancers than smaller cancers. If the $\mathrm{x}$ - and $\mathrm{y}$-axes were reversed for the hypothesis that thyroid cancers led to CT scans, there would be less CT scans for a given number of larger nonpapillary cancers than smaller nonpapillary cancers. The opposite would be expected for large invasive anaplastic and medullary cancers-large tumors are more likely to require CT for evaluation of local invasion. Thus, it is unlikely that CT work-up is the major explanation for the relationship between CT scans and thyroid cancer.

A less likely alternative explanation for the linear relationship between thyroid cancer and CT is that radiation exposure from CT scanning is causing thyroid cancer. The mean radiation dose to the thyroid from a CT scan of the neck ranges from 17-34 mGy, which is within the range that may increase the risk for thyroid cancer in children. ${ }^{18-20}$ However, most people receiving neck CT scans are older adults, and the risk for cancer from radiation exposure decreases sharply with increasing age. ${ }^{21}$ Pooled analyses of studies of thyroid cancer in patients exposed to radiation found that there was little risk for excess cancer when exposure was after age 20 years. $^{22}$ The International Commission on Radiological Protection also estimates that the relative risk for thyroid cancer decreases by $57 \%$ for each decade of life. ${ }^{23}$ Patients having a CT scan of the cervical spine are often in a younger age group, but they are still mostly adults. In a large multicenter cervical spine trauma study, the mean age of patients having cervical spine imaging for trauma was 37 years. ${ }^{24}$ Furthermore, radiation-induced malignant disease occurs decades after the radiation exposure. Kikuchi et $\mathrm{al}^{25}$ studied patients with therapeutic radiation exposure to the neck and found that the mean latency period for thyroid cancer was 28 years. Thus, the more dramatic increase in CT scans in the US population that has occurred in the last 10 years should not affect the incidence of thyroid cancer until future years.
Our study had several limitations. First, the SEER data base did not provide information on how the tumors were diagnosed. The impact of other potential sources of occult papillary microcarcinomas, such as thyroidectomies for benign indications or a change in pathologic technique or classification, cannot be measured for these data. ${ }^{26,27}$ Second, the data bases for thyroid cancer and CT volume were not exactly matched. The patients in the SEER data base represent $10 \%$ of the US population, whereas the NCRP CT data base is for the entire US population. Third, it was not possible to exclude CT studies that do not image the thyroid from the NCRP CT data base. To use the available data, we assumed that the proportion of studies that included the neck had increased at the same rate with time. In addition, we could not exclude repeated studies performed in the same patient or multiple scans performed in the same session. These 2 limitations of the NCRP data base could be overcome by obtaining CT use data from the Medicare data base, but the Medicare data base only includes patients 65 years and older and would not account for correlation with younger patients. This would have been a major limitation because our results found that the greatest growth in thyroid cancer incidence was seen in the 50- to 64-year-old group.

Although our analysis cannot prove causation, this relationship may help to focus future work on testing the hypothesis that CT scans contribute to the increased detection of incidental thyroid nodules leading to an increased incidence in thyroid cancer. Until then, we should improve on current practices by developing evidence-based and cost-effective guidelines for the work-up of incidental thyroid nodules seen on CT. Currently thyroid nodules are seen in 1 in 6 CT neck studies. ${ }^{21,28}$ The radiologist's approach to reporting incidental thyroid nodules on $\mathrm{CT}$ can vary because CT cannot differentiate between a benign and malignant nodule, and there is a lack of clear guidelines for work-up. ${ }^{21,29}$

\section{CONCLUSIONS}

The incidence of subcentimeter papillary carcinoma is growing at an exponential rate without significant change in mortality rate. There is a very strong linear relationship between new cases of subcentimeter papillary carcinomas and the number of CT scans per year. This trend suggests that an increase in CT scans may increase the detection of incidental thyroid cancers.

Disclosures: Gary Lyman—UNRELATED: Grants/Grants Pending: Amgen, ${ }^{*}$ Comments: $\mathrm{PI}$ on research grant to Duke University. *Money paid to institution.

\section{REFERENCES}

1. Davies L, Welch HG. Increasing incidence of thyroid cancer in the United States, 1973-2002. JAMA 2006;295:2164-67

2. Sandhu GS, Andriole GL. Overdiagnosis of prostate cancer. J Natl Cancer Inst Monogr 2012;2012:146-51

3. McLeod DS, Sawka AM, Cooper DS. Controversies in primary treatment of low-risk papillary thyroid cancer. Lancet 2013;381:1046-57

4. Etzioni R, Penson DF, Legler JM, et al. Overdiagnosis due to prostate-specific antigen screening: lessons from U.S. prostate cancer incidence trends. J Natl Cancer Inst 2002;94:981-90

5. Frates MC, Benson CB, Charboneau JW, et al. Management of thyroid nodules detected at US: Society of Radiologists in Ultrasound consensus conference statement. Radiology 2005;237:794-800

6. Cooper DS, Doherty GM, Haugen BR, et al. Revised American Thyroid Association management guidelines for patients with thyroid 
nodules and differentiated thyroid cancer. Thyroid 2009;19: $1167-214$

7. Matin A, Bates DW, Sussman A, et al. Inpatient radiology utilization: trends over the past decade. AJR Am J Roentgenol 2006;186:7-11

8. Rao VM, Levin DC, Parker L, et al. Trends in utilization rates of the various imaging modalities in emergency departments: nationwide Medicare data from 2000 to 2008. J Am Coll Radiol 2011;8:706-09

9. Broder J, Warshauer DM. Increasing utilization of computed tomography in the adult emergency department, 2000-2005. Emerg Radiol 2006;13:25-30

10. National Council on Radiation Protection and Measurements., National Council on Radiation Protection and Measurements. Scientific Committee 6-2 on Radiation Exposure of the U.S. Population. Ionizing Radiation Exposure of the Population of the United States. Bethesda, MD: National Council on Radiation Protection and Measurements; 2009

11. Hughes DT, Haymart MR, Miller BS, et al. The most commonly occurring papillary thyroid cancer in the United States is now a microcarcinoma in a patient older than $\mathbf{4 5}$ years. Thyroid 2011; 21:231-36

12. Pellegriti G, Frasca F, Regalbuto C, et al. Worldwide increasing incidence of thyroid cancer: update on epidemiology and risk factors. $J$ Cancer Epidemiol 2013;2013:965212

13. Moore CL, Copel JA. Point-of-care ultrasonography. N Engl J Med 2011;364:749-57

14. Kim DW, Kim SH, Jung SJ. Successful sonography-guided fine-needle aspiration biopsy of a 1-millimeter-diameter papillary thyroid microcarcinoma. AJNR Am J Neuroradiol 2010;31:1082-84

15. Chen AY, Jemal A, Ward EM. Increasing incidence of differentiated thyroid cancer in the United States, 1988-2005. Cancer 2009; 115:3801-07

16. Wiest PW, Hartshorne MF, Inskip PD, et al. Thyroid palpation versus high-resolution thyroid ultrasonography in the detection of nodules. J Ultrasound Med 1998;17:487-96

17. Hobbs $\mathrm{H}, \mathrm{Bahl} \mathrm{M}$, Nelson RC, et al. Incidental thyroid nodules detected on imaging: can workup be reduced by using the SRU recommendations and the 3-tier system? AJR Am J Roentgenol 2014. In press.
18. Hoang JK, Yoshizumi TT, Nguyen G, et al. Variation in tube voltage for adult neck MDCT: effect on radiation dose and image quality. AJR Am J Roentgenol 2012;198:621-27

19. Leswick DA, Hunt MM, Webster ST, et al. Thyroid shields versus $\mathrm{z}$-axis automatic tube current modulation for dose reduction at neck CT. Radiology 2008;249:572-80

20. Mazonakis M, Tzedakis A, Damilakis J, et al. Thyroid dose from common head and neck CT examinations in children: is there an excess risk for thyroid cancer induction? Eur Radiol 2007; 17:1352-57

21. Nguyen XV, Choudhury KR, Eastwood JD, et al. Incidental thyroid nodules on CT: evaluation of 2 risk-categorization methods for work-up of nodules. AJNR Am J Neuroradiol 2013;34:1812-17

22. Ron E, Lubin JH, Shore RE, et al. Thyroid cancer after exposure to external radiation: a pooled analysis of seven studies. Radiat Res 1995;141:259-77

23. The 2007 Recommendations of the International Commission on Radiological Protection. ICRP publication 103. Ann ICRP 2007; 37:1-332

24. Hoffman JR, Mower WR, Wolfson AB, et al. Validity of a set of clinical criteria to rule out injury to the cervical spine in patients with blunt trauma. National Emergency X-Radiography Utilization Study Group. N Engl J Med 2000;343:94-99

25. Kikuchi S, Perrier ND, Ituarte P, et al. Latency period of thyroid neoplasia after radiation exposure. Ann Surg 2004;239:536-43

26. Salajegheh A, Petcu EB, Smith RA, et al. Follicular variant of papillary thyroid carcinoma: a diagnostic challenge for clinicians and pathologists. Postgrad Med J 2008;84:78-82

27. de Matos LL, Del Giglio AB, Matsubayashi CO, et al. Expression of CK-19, galectin-3 and HBME-1 in the differentiation of thyroid lesions: systematic review and diagnostic meta-analysis. Diagn Pathol 2012;7:97

28. Yousem DM, Huang T, Loevner LA, et al. Clinical and economic impact of incidental thyroid lesions found with CT and MR. AJNR Am J Neuroradiol 1997;18:1423-28

29. Shetty SK, Maher MM, Hahn PF, et al. Significance of incidental thyroid lesions detected on CT: correlation among CT, sonography, and pathology. AJR Am J Roentgenol 2006;187:1349-56 